\title{
Electronic structure of the self-trapped exciton in sodium chloride
}

\author{
A M Stoneham \\ Theoretical Physics Division, AERE, Harwell, Didcot, Berks
}

Received 5 March 1974

\begin{abstract}
The electronic structure of the relaxed exciton in sodium chloride is investigated using a Hartree-Fock method. The model concentrates on a cluster of two sodium and two chlorine ions surrounded by an array of point charges. The excited states of the exciton and the associated transitions are discussed. The results are compared with experiment and with the phenomenological models proposed by earlier workers. A number of features are confirmed, giving a general picture close to that of Kabler and his co-workers. One problem emerges, since it proves possible to understand the $\sigma$ luminescence or the $\pi$ luminescence, but not both at present. Possible reasons are discussed. In other respects, there is good agreement with experiment, especially for the optical transitions starting from the lowest triplet state of the exciton.
\end{abstract}

\section{Introduction}

It has been known for some years that substantial lattice distortion follows the creation of an exciton in the alkali halides. The present work is concerned with the electronic structure of the relaxed exciton in the distorted lattice configuration. The theory of this centre should be important for several reasons. Thus it can help in the interpretation of some of the ambiguous features of the experiments, such as the identification of certain of the states involved when there is more than one alternative. It also provides a check of the various theoretical techniques needed in the analysis of the mechanism of formation of colour centres.

The theory of the exciton is currently being studied by three methods: the selfconsistent, open-shell Hartree-Fock method discussed here, a semi-empirical molecular orbital method, and a pseudopotential approach for the excitonic electron. All have particular advantages for different features, and it is hoped that the combination of these methods will permit treatment of all the important features. The present work uses a program ATMOL, provided by Dr V R Saunders of the Atlas Laboratory. This program does self-consistent open-shell Hartree-Fock calculations on molecular systems. Like all such programs, there is a severe limit on the number of electrons which can be treated (here 68), so that approximations are needed in treating solid state problems. The restriction also means that only the lighter alkali halide system can be tackled. Here we describe work on $\mathrm{NaCl}$ only. Some brief comments on $\mathrm{LiF}$ will be made, but a full discussion of the fluorides $\mathrm{LiF}$ and $\mathrm{NaF}$ will be deferred till a later paper. The $\mathrm{NaCl}$ system is treated first because there are so many experimental results available.

The extensive experiments on the relaxed exciton lead to the following main conclusions. First, the exciton can best be regarded as an electron bound to a self-trapped 
hole (Kabler 1964, Murray and Keller 1965). In the self-trapped hole (or $V_{k}$ centre) the hole is shared between two halogens, so that two $\mathrm{X}^{-}$ions are replaced by $\mathrm{X}_{2}^{-}$at a somewhat smaller separation. Second, there are two components in the luminescence from exciton recombination in most alkali halides. One is spin-allowed, has a short lifetime, and is $\sigma$ polarized, with dipole vector parallel to the $\mathrm{X}_{2}^{-}$axis. The other occurs at lower energy, with $\pi$ polarization; it has a long lifetime, apparently because it results from an initial spin triplet state and is spin-forbidden. Models discussing the nature of these states have been discussed by Wood (1966), Kabler and Patterson (1967). Fuller et al (1970) and Blair et al (1972). Whilst there are differences, it seems agreed that the exciton wavefunction is largely confined to a small group of atoms: the two halogens which constitute the $V_{k}$ centre, and their two mutual neighbouring cations. There is experimental evidence for this localization, for the long lifetime of the triplet state has been exploited for both spin-resonance and optical studies (Marrone et al 1973, Wasiela et al 1973, Fisbach et al 1973. Fowler et al 1973). It is this local localization which makes possible the Hartree-Fock calculations of the type reported here.

\section{The model}

The two most important features in defining the model are its geometry and the choice of basis orbitals. The geometry involves several aspects: which ions are treated in detail, which are regarded as point charges, and the extent of any lattice distortion assumed.

\subsection{Geometry}

In the present model four ions are treated in detail, a group (MX) $)_{2}$ of two halogens X and two alkalis $M$. These are surrounded by 44 point charges. The array is shown in figure 1 ; in essence there are three $4 \times 4$ squares of ions, with the ions treated in detail in the middle of the central layer.

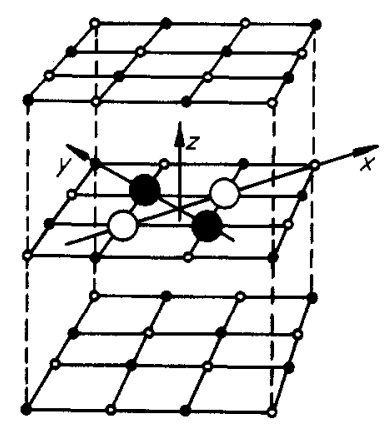

Figure 1. Geometry of cluster treated. The open circles represent halogens, the full circles alkalis. The large circles indicate the four ions treated in detail; other ions shown were treated as point charges. For clarity, the scale of the $z$ axis has been expanded in the figure : the three planes were held at the correct nearest-neighbour distance during the calculations.

The number of point changes surrounding the central $(\mathrm{MX})_{2}$ cluster is a compromise. Obviously, the more charges, the better the full crystal is modelled. But equally, the more charges, the longer the computation time. In the present grouping all the first, 
second and third neighbours of the cluster ions are present, so each ion in the $(\mathrm{MX})_{2}$ treated in detail has an environment very similar to that in the full crystal. The pointcharge configuration also contains equal numbers of anions and cations. This proves important in estimates of ionization energies.

In treating only the four $(\mathrm{MX})_{2}$ ions in detail, we have been guided by experiment. The work of Kabler and his co-workers suggests that almost all the properties of the relaxed exciton can be described in terms of the $\left(\mathrm{X}^{-}\right)_{2}$ molecular ion, with some qualifications associated with the possibly diffuse electron part of the wavefunction. Blair et al, however, have stressed that the excited states involve the nearest metal ions. They also suggest that the weakness of the $\pi$ transition may involve the parity selection rule as well as a spin selection rule. The detailed discussion of the (MX) $)_{2}$ complex encompasses both models. Indeed, one aim of the present work is to resolve some of the unsettled questions concerning the two interpretations. The ATMOL program also imposes limits on the number of electrons which can be treated in detail. For an $(\mathrm{MX})_{2}$ complex, calculations are only practical for the lighter elements, $\mathrm{Li}, \mathrm{Na}, \mathrm{F}$ and $\mathrm{Cl}$. We shall stress the $\mathrm{NaCl}$ results here, since both lithium salts and fluorides can show behaviour atypical of the other alkali halides.

\subsection{Lattice relaxation}

The next question concerns the lattice relaxation near the exciton. The analysis of transition energies by Kabler and his co-workers suggests that the two halogen ions have almost exactly the same configuration as in the $V_{k}$ centre. The present calculations put all ions and point charges at their positions in the perfect lattice except the two halogens, which are moved to match the $\mathrm{V}_{\mathrm{k}}$ configuration. It is then a matter of deciding what halogen separation occurs in the $V_{k}$ centre. This has been done empirically using a model of the $V_{k}$ centre as an $X_{2}^{-}$molecular ion whose separation alone has been changed. The optical transition energies and hyperfine constants are then known as a function of halogen separation (Jette et al 1969, Gilbert and Wahl 1971). The separation adopted gives the best overall fit to the observed transition energies and spin resonance data. Values found for the halogen-halogen separation are:

$$
\begin{array}{ll}
\mathrm{LiF} & 3.75 \mathrm{au} \\
\mathrm{NaF} & 3.75 \mathrm{au} \\
\mathrm{NaCl} & 5.0 \mathrm{au} .
\end{array}
$$

Other prescriptions are possible, but seem to give very similar values (Dr M N Kabler, private communication). Less detailed calculations have been made for a variety of other separations, including the values for the perfect crystal structure.

It is possible, but extravagant, to use this Hartree-Fock approach to investigate the exciton properties for a wide range of geometries. These geometries should include the configurations relevant to studies of the radiation damage process as well as the obvious symmetrical motion of the cations in the $(\mathrm{MX})_{2}$ complex. It is more efficient to adopt the strategy currently in progress at Harwell, namely to use the Hartree-Fock calculations for special configurations only. Properties for other configurations can be best obtained by more approximate methods (like the CNDO approach) which have been checked against the more detailed calculations.

In passing, it should be noted that the lattice distortion is the main reason that an $(\mathrm{MX})_{2}$ cluster is adequate. The electron and hole wavefunctions in an undistorted lattice are probably much more extended. Thus the present model, as it stands, may not give good band structures for the perfect crystal. 


\subsection{Basis orbitals}

The basis orbitals chosen have been those of Clementi (1965) for the various alkali and halogen atoms. These are listed in table 1 . The ATMOL program adjusts the weights of the different terms listed, without changes in the exponents. In the present calculation,

\begin{tabular}{|c|c|c|c|c|}
\hline & $\mathrm{Li}$ & $\mathrm{Na}$ & $\mathrm{F}$ & $\mathrm{Cl}$ \\
\hline \multirow[t]{2}{*}{ 1s } & $2 \cdot 433$ & $9 \cdot 715$ & $7 \cdot 918$ & 12.059 \\
\hline & $4 \cdot 518$ & $13 \cdot 147$ & 11.011 & 17.650 \\
\hline \multirow[t]{2}{*}{$2 \mathrm{~s}$} & 0.450 & $2 \cdot 604$ & 11.947 & 4.926 \\
\hline & 0.850 & $3 \cdot 910$ & 3.096 & 6.983 \\
\hline \multirow[t]{2}{*}{$3 \mathrm{~s}$} & - & 0.755 & 1.845 & 2.009 \\
\hline & & 1.259 & $\begin{array}{l}4 \cdot 171 \\
(2 \cdot 50) \\
(0 \cdot 35)\end{array}$ & $3 \cdot 342$ \\
\hline \multirow[t]{2}{*}{$2 p$} & 一 & $2 \cdot 571$ & & $5 \cdot 357$ \\
\hline & & $\begin{array}{l}5.496 \\
(1.0)\end{array}$ & & $9 \cdot 567$ \\
\hline \multirow[t]{2}{*}{$3 p$} & - & $(0.53)$ & & 1.609 \\
\hline & & $(2 \cdot 0)$ & & 2.859 \\
\hline
\end{tabular}

another ATMOL facility has been used: each of the Slater-type basis orbitals was approximated by a sum of three gaussian orbitals. This speeded the calculation to some extent, removed all problems with three- and four-centre integrals, and has apparently little effect on the accuracy of the results.

The atomic basis chosen should be adequate for the low-lying excitations of the $(\mathrm{MX})_{2}$ complex. We discuss later those features for which the present basis may not be adequate, and the way in which such features may be examined.

\section{The relaxed exciton in $\mathrm{NaCl}$}

The results for $\mathrm{NaCl}$ will now be summarized and compared with experiment. In all cases the results quoted are from self-consistent open-shell calculations: Koopmans' approximation has not been used. It has been found that differences of one-electron eigenvalues give a very poor description of the transition energies. This is especially so when the occupancies of the chlorine $3 p$ orbitals are changed.

\subsection{The ground states of the exciton and the $V_{k}$ centre}

The ground state of the exciton differs only from the ground state of the perfect crystal through the lattice distortion assumed (and, of course, through approximations we have made). Its properties are calculated both to identify the order of the one-electron levels and to obtain an energy zero for the various transitions.

For the exciton ground state, calculation confirms that the ionic state is lowest in energy. The system then contains $\mathrm{Na}^{+}$ions, with full $1 \mathrm{~s}, 2 \mathrm{~s}$ and $2 \mathrm{p}$ shells, and $\mathrm{Cl}^{-}$ions 
with full $1 \mathrm{~s}, 2 \mathrm{~s}, 2 \mathrm{p}, 3 \mathrm{~s}$ and $3 \mathrm{p}$ orbitals. In order of increasing energy, the groups are:

$$
\begin{gathered}
E\left(\mathrm{Cl}^{-} 1 \mathrm{~s}\right)<E\left(\mathrm{Na}^{+} 1 \mathrm{~s}\right)<E\left(\mathrm{Cl}^{-} 2 \mathrm{~s}\right)<E\left(\mathrm{Cl}^{-} 2 \mathrm{p}\right)<E\left(\mathrm{Na}^{+} 2 \mathrm{~s}\right)<E\left(\mathrm{Na}^{+} 2 \mathrm{p}\right) \\
<E\left(\mathrm{Cl}^{-} 3 \mathrm{~s}\right)<E\left(\mathrm{Cl}^{-} 3 \mathrm{p}\right) .
\end{gathered}
$$

For practical purposes the overlap of the ions only removes significantly the degeneracy of the $\mathrm{Cl}^{-} 3 \mathrm{p}$ orbitals. Thus the spread of the $\mathrm{Na}^{+} 2 \mathrm{p}$ levels, for example, is only about $0.15 \mathrm{eV}$. The $\mathrm{Cl}^{-} 3 \mathrm{p}$ levels are, however, appreciably split. The order of the one-electron levels is :

$$
E\left(\mathrm{~A}_{1 \mathrm{~g}}\right)<E\left(\mathrm{~B}_{1 \mathrm{u}}\right)<E\left(\mathrm{~B}_{2 \mathrm{u}}\right)<E\left(\mathrm{~B}_{1 \mathrm{~g}}\right)<E\left(\mathrm{~B}_{2 \mathrm{~g}}\right)<E\left(\mathrm{~B}_{3 \mathrm{u}}\right)
$$

in increasing order, and the spread of the one-electron eigenvalues is about $3.7 \mathrm{eV}$. The notation for the orbitals is discussed in the Appendix, and corresponds to the choice of Blair et al.; the relation to the notation of Kabler and co-workers is also described. Whilst these eigenstates are constructed primarily from $\mathrm{Cl}^{-} 3 \mathrm{p}$ states, there is also a small admixture of other anion and cation functions.

The total energy of the ground state proves to be -1240.49602 atomic units. In all future results, energy differences alone will be quoted. They can be converted to absolute energies, if necessary, using this figure.

In the $\mathrm{V}_{\mathrm{k}}$ centre, one electron is removed, and $\mathrm{sCF}$ calculation repeated. The new ground state is higher in energy by $9.38 \mathrm{eV}$. This energy is an estimate of the ionization energy of the complex. It is similar in magnitude to Roessler and Walker's (1968) experimental estimate of $8.97 \mathrm{eV}$ for the band gap in $\mathrm{NaCl}$, although the figures are not strictly comparable because of the different lattice configurations.

The electronic structure and excitations of the $V_{k}$ centre will be discussed in more detail in a subsequent paper.

\subsection{Singlet excited states of the exciton}

The singlet excited states are important because they determine the spin-allowed transitions of the exciton, including the highly-allowed $\sigma$ transition observed at $5.4 \mathrm{eV}$. The excited states are generated by transferring an electron from one of the upper occupied levels to one of the lower unoccupied levels, and letting the program continue to self-consistency in the new state. Table 2 summarizes the results obtained.

The upper occupied levels are primarily $\mathrm{Cl}^{-} 3 \mathrm{p}$ orbitals, and were described in $\S 3.1$. The highest one, with $\mathrm{B}_{3 u}$ symmetry, contains very small admixtures of $\mathrm{Cl}^{-} 3 \mathrm{~s}$ and $\mathrm{Na}^{+} 2 \mathrm{p}$. The important unoccupied one-electron levels, in order of increasing energy, are

(a) $\mathrm{A}_{1 \mathrm{~g}}$, primarily consisting of $\mathrm{Na}^{+} \mathrm{s}$ orbitals, with a significant $\mathrm{Cl}^{-} \mathrm{s}$ admixture, but very small $\mathrm{Na}^{+} \mathrm{p}$ and $\mathrm{Cl}^{-} \mathrm{p}$ contributions :

(b) $\mathrm{B}_{1 \mathrm{u}}$, primarily consisting of $\mathrm{Na}^{+} \mathrm{s}$ orbitals, but with a significant $\mathrm{Cl}^{-} \mathrm{p}$ contribution. There is a small $\mathrm{Na}^{+} \mathrm{p}$ admixture:

(c) $\mathrm{A}_{1 \mathrm{~g}}$, again primarily involving $\mathrm{Na}^{+} \mathrm{s}$ orbitals. There is a significant $\mathrm{Cl}^{-} \mathrm{p}$ fraction, and there are small $\mathrm{Na}^{+} \mathrm{p}$ and $\mathrm{Cl}^{-} \mathrm{s}$ admixtures.

It is probable that this second $A_{1 \mathrm{~g}}$ state is unbound, and it will not be discussed further. Note that the $A_{1 \mathrm{~g}}$ orbital lies below $\mathbf{B}_{1 \mathrm{u}}$, contrary to the model proposed by Blair et al.

The lowest-energy excitation involves the $B_{3 u} \leftrightarrow A_{1 g}$ transition. The transition energy is predicted to be $6.25 \mathrm{eV}$. In many aspects the transition describes the observed 
$\sigma$-luminescence well. The energy is quite good $(6.25 \mathrm{eV}$, compared with $5.4 \mathrm{eV})$, the symmetry is right, and the initial and final wavefunctions appear to have sufficient overlap to explain the high observed transition probabilities. The problems arise when one compares the $\sigma$ and $\pi$ transitions, for the $\pi$ transition is spin-forbidden, starting from a triplet state, and occurs at the lower energy of $3.4 \mathrm{eV}$. In $\S 3.3$ we shall see that the $\pi$ luminescence is described rather well by the assumption that the initial state is the

Table 2. Summary of transition energies in $\mathrm{NaCl}$. The states and transitions are labelled by $|X ; Y\rangle$ in which, relative to the singlet ground state, electrons have been removed from $Y$ and placed in orbitals $X$. Energies are in $\mathrm{eV}$.

\begin{tabular}{|c|c|c|c|c|}
\hline \multirow{2}{*}{ Excitation } & \multicolumn{2}{|l|}{ States } & \multirow{2}{*}{$\begin{array}{l}\text { Energy } \\
\text { (theory) }\end{array}$} & \multirow{2}{*}{ Experiment } \\
\hline & Initial & Final & & \\
\hline $\begin{array}{l}\text { Singlet states possibly } \\
\text { related to } \sigma \text { luminescence }\end{array}$ & $\mid \begin{array}{l}\left.\alpha \mathbf{A}_{1 \mathrm{~g}}: \alpha \mathbf{B}_{3 u}\right\rangle \\
\left|\alpha \mathbf{B}_{1 \mathrm{u}} ; \alpha \mathbf{B}_{3 u}\right\rangle\end{array}$ & $|:\rangle$ & $\begin{array}{l}6 \cdot 25 \\
7 \cdot 73\end{array}$ & $\begin{array}{l}5 \cdot 4 \\
(\sigma \text { luminescence })\end{array}$ \\
\hline $\begin{array}{l}\text { Lowest triplet } \\
\text { (for } \pi \text { luminescence) }\end{array}$ & $\left|\beta \mathrm{A}_{1 \mathrm{~g}} ; \alpha \mathrm{B}_{3 \mathrm{u}}\right\rangle$ & $|:\rangle$ & 6.22 & $\begin{array}{l}3.4 \\
(\pi \text { luminescence })\end{array}$ \\
\hline Singlet-triplet splitting & $\left|\beta \mathrm{A}_{1 \mathrm{~g}} ; \beta \mathrm{B}_{3 \mathrm{u}}\right\rangle$ & $\left|\alpha \mathbf{A}_{1 \mathrm{~g}} ; \beta \mathbf{B}_{3 \mathrm{u}}\right\rangle$ & 0.03 & 0.04 \\
\hline $\begin{array}{l}\mathrm{V}_{\mathrm{k}} \text {-like excitations of } \\
\text { triplet. Final hole orbital } \\
\text { given only }\end{array}$ & $\begin{array}{l}X \equiv \mathrm{A}_{1 \mathrm{~g}}(\sigma) \\
X \equiv \mathrm{B}_{1 \mathrm{~g}}(\pi y) \\
X \equiv \mathrm{B}_{2 \mathrm{~g}}(\pi z)\end{array}$ & & $\left.\begin{array}{l}3.95 \\
1.95 \\
1.79\end{array}\right\}$ & $\begin{array}{l}3 \cdot 6 \\
(2 \cdot 1)\end{array}$ \\
\hline M-like excitation of triplet & $\left|\beta A_{1 g} ; \alpha B_{3 u}\right\rangle$ & $\left|\beta \mathbf{B}_{1 u} ; \alpha \mathbf{B}_{3 u} ;\right\rangle$ & $1 \cdot 49$ & $2 \cdot 1$ \\
\hline Ionization of triplet & $\left|\beta A_{1 g}: \alpha B_{3 u}\right\rangle$ & $\left|: \alpha B_{3 u}\right\rangle$ & $3 \cdot 16$ & $2 \cdot 8$ \\
\hline Ionization of singlet & $\left|\alpha \mathbf{A}_{18} ; \alpha \mathbf{B}_{3 u}\right\rangle$ & $\left|: x B_{3 u}\right\rangle$ & $9 \cdot 38$ & \\
\hline
\end{tabular}

spin triplet state obtained by the $B_{3 \mathrm{u}} \leftrightarrow \mathrm{A}_{1 \mathrm{~g}}$ transition. All would be well if the singlettriplet splitting were as large as $1.9 \mathrm{eV}$. But detailed calculations show that this splitting is smaller by about a factor 50 , and that it cannot be increased by changing the geometry. Nor are there any signs of a different equilibrium lattice configuration for the singlet and triplet states, for their energies vary in the same way when the $\mathrm{Cl}-\mathrm{Cl}$ separation is varied.

The problem has not yet been resolved. One possibility is that the $\sigma$ transition originates from a higher singlet state. If so, then one must explain why the exciton does not show luminescence from the lowest singlet state; presumably the answer, if any, lies in the kinetics of the exciton formation, rather than in the energetics. One must also identify the higher singlet state. There are then three main possibilities. One is that a higher unoccupied level is involved. The obvious one transfers an electron from the $B_{3 u}$ orbital to the unoccupied $B_{1 u}$ orbital. The transition energy, $7.73 \mathrm{eV}$, is larger than the triplet transition energy $(\$ 3.3)$ by $1.51 \mathrm{eV}$, similar to the observed $1.9 \mathrm{eV}$. But the state has the wrong symmetry. This objection can also be raised to the second possibility, that the electron is transferred from a different orbital than $\mathbf{B}_{3 \mathrm{u}}$. The third possibility is that a crucial state is omitted because of the limitations of the basis chosen. We return to this later.

\subsection{Triplet states: the lowest state and its luminescence}

The triplet excited states are of special interest because of the amount of experimental 
information available. Spin-resonance data have been obtained by Wasiela et al (1973) and Marrone et al (1973), and Fuller et al (1970) and Williams and Kabler (1974) have studied the optical excitations of the triplet state. The reason that this information is accessible is, of course, the long lifetime of the triplet because its decay is spin-forbidden.

The lowest triplet state results from the transition of an electron with $\alpha$ spin in a $B_{3 u}$ orbital to an unoccupied $\mathrm{A}_{1 \mathrm{~g}}$ orbital with $\beta$ spin. The transition energy predicted is $6.22 \mathrm{eV}$, somewhat larger than the observed $3.4 \mathrm{eV}$. The discrepancy could result from our assumption that the $\mathrm{Na}^{+}$ions are at their perfect lattice positions, or from some other approximation. The singlet-triplet splitting for this excited state is about $0.03 \mathrm{eV}$, close to that of $0.04 \mathrm{eV}$ deduced from experiment for the higher $\pi$-states by Fowler $e$ al (1973). The ionization energy of the triplet state, predicted at $3.16 \mathrm{eV}$, is also close to experiment, for Williams and Kabler estimate $2.8 \mathrm{eV}$ from their optical spectroscopy.

The triplet luminescence occurs because the spin-orbit coupling renders the spinforbidden transition slightly allowed (Kabler and Patterson 1962). This can be described by involving another excited state, and the obvious question is which excited state is important? The state is most easily identified if we introduce a notation $|X ; Y\rangle$. Here $X^{\prime}$ denotes the orbitals which are occupied in the state in question, but unoccupied in the singlet ground state, and $Y$ denotes the orbitals occupied in the singlet ground state but currently unoccupied. Thus the lowest triplet state is $\left|\beta \mathbf{A}_{1 \mathrm{~g}} ; \alpha \mathbf{B}_{3 \mathrm{u}}\right\rangle$, for example, and the singlet ground state is $|;\rangle$. The matrix element for the $\pi$ luminescence from the lowest triplet state involves $\Omega_{1}$, the electric dipole operator normal to the $\mathrm{Cl}-\mathrm{Cl}$ axis of the exciton, and $\Omega_{2}$, the spin-orbit coupling of the halogen $3 p$ electrons. There are two contributions to the matrix element, which has the form:

$$
\left\langle\beta \mathrm{A}_{1 \mathrm{~g}} ; \alpha \mathrm{B}_{3 \mathrm{u}}\left|\Omega_{1}\right| I\right\rangle\left\langle I\left|\Omega_{2}\right|:\right\rangle / \Delta E_{I}+\left\langle\beta \mathrm{A}_{1 \mathrm{~g}} ; \alpha \mathbf{B}_{3 \mathrm{u}}\left|\Omega_{2}\right| J\right\rangle\left\langle J\left|\Omega_{1}\right| ;\right\rangle / \Delta E_{J} .
$$

In the first term, the intermediate state is $|I\rangle \equiv\left|\beta \mathrm{B}_{1 \mathrm{u}}: \alpha \mathrm{B}_{3 \mathrm{u}}\right\rangle$ which is an $\mathrm{M}$-like excitation of the exciton ( $\mathrm{cf} \& 3.4$ ), and $\Delta E_{I}$ is estimated to be $1.49 \mathrm{eV}$. In the second term the intermediate state is $|J\rangle \equiv\left|\beta \mathrm{A}_{1 \mathrm{~g}} ; \beta \mathrm{B}_{1 \mathrm{u}}\right\rangle$, a $\mathrm{V}_{\mathrm{k}}$-like excitation of the sense of $\S 3.4$. Detailed comparisons of these terms have not been made yet. Probably the second term dominates for, whilst $\Delta E_{J}$ is about twice $\Delta E_{I}$, there are a variety of overlap factors which favour the term in $|J\rangle$.

\subsection{Excited triplet states}

As observed by Williams and Kabler, there are two classes of excited state $|X: Y\rangle$ obtained by optical excitation of the lowest tripler state $\left|\beta \mathrm{A}_{1 \mathrm{~g}} ; \alpha \mathrm{B}_{3 \mathrm{u}}\right\rangle$. On the one hand there are the $\mathrm{V}_{\mathbf{k}}$-like transitions, in which the hole in the $\mathrm{Cl}^{-} 3 \mathrm{p}$ orbitals changes state :

$$
\left|\beta \mathrm{A}_{1 \mathrm{~g}} ; \alpha \mathrm{B}_{3 \mathrm{u}}\right\rangle \rightarrow\left|\beta \mathrm{A}_{1 \mathrm{~g}}: \alpha Y\right\rangle \text {. }
$$

On the other hand, there are transitions of the excited electron:

$$
\left|\beta \mathrm{A}_{1 \mathrm{~g}} ; \alpha \mathrm{B}_{3 \mathrm{u}}\right\rangle \rightarrow\left|\beta X ; \alpha \mathrm{B}_{3 \mathrm{u}}\right\rangle .
$$

The transitions in this second group are roughly analogous to those of the excitations of the $\mathrm{M}$ centre. Our calculations include the optically allowed transitions of both types. In describing the states, we shall simply identify the states by $X$ and $Y$ from the above expressions.

There are three $\mathrm{V}_{\mathbf{k}}$-like transitions : $\mathrm{a} \sigma$ transition $\left(Y=\mathrm{A}_{1 \mathrm{~g}}\right)$ and two nearly-degenerate $\pi$-transitions $\left(Y=\mathbf{B}_{1 \mathrm{~g}}, \mathbf{B}_{2 \mathrm{~g}}\right.$ ) split by the crystal field. The $\sigma$ transition is at $3.95 \mathrm{eV}$, close to the observed $3.6 \mathrm{eV}$. The $\pi$ transitions are at $1.79 \mathrm{eV}\left(\mathrm{B}_{2 \mathrm{~g}}\right)$ and $1.95 \mathrm{eV}\left(\mathrm{B}_{1 \mathrm{~g}}\right)$, 
close to the band at $2 \cdot 1 \mathrm{eV}$ which Williams and Kabler attribute to M-like transitions of the excited electron.

Only one $M-$ like transition occurs within a reasonable range of energies ( $X \equiv \mathrm{B}_{1 \mathrm{u}}$ ), and this is a $\pi$ transition at $1.49 \mathrm{eV}$. Again, this is not far from the $2.1 \mathrm{eV}$ band observed. The limitations of our choice of basis are particularly apparent here, for no $\sigma$ transitions are predicted for energies lower than the ionization energy of the exciton. That such transitions should occur is implied by a theorem of Mott and Gurney $(1948, \S 5.1)$.

\subsection{Adequacy of basis}

There are two reasons for questioning the adequacy of the basis chosen. On the one hand, there is an apparent contradiction between the singlet and triplet states. Either can be explained straightforwardly, but not both. On the other hand, it is clear that we have omitted a number of excited states of the electron bound to the hole in its lowest state, the so-called M-like states of Williams and Kabler. Before discussing possible basis modifications, it should be stressed that it is not trivial to increase the basis substantially: the present calculations already work very close to the limits of the ATMOL program. Thus extensions must be by special tricks or by use of other methods.

The possible extensions fall into three broad classes. First, it may be that more atoms than the four considered in detail play an important rôle in the relaxed exciton. If so, work currently in progress using the semi-empirical molecular-orbital method CNDO/2 should show the desired features. This approach is also particularly suited to a study of the radiation damage processes associated with the bound exciton. Second, if highlyexcited states of the electron are involved, it may be that an atomic orbital basis is not convenient. If so, a suitable approach (again currently in progress) should be to use defect-centred electron functions and a development of the pseudopotential approach of Bartram et al (1968). The third possibility is that there are important states which would appear when an extended (or modified) atomic basis was used. One might feel, for instance, that more $\mathrm{s}$ or $\mathrm{p}$ orbitals were needed on the $\mathrm{Cl}$ or $\mathrm{Na}$, or that the chlorine $\mathrm{d}$ orbitals were important. Data for the free atom suggest this is possible, but not especially plausible. Results using a somewhat extended basis for LiF do not add much support to this possibility either. Moreover, halogen s orbitals have the wrong symmetry to help in the singlet-triplet problem. But this third possibility cannot be ruled out without larger calculations than are possible at present.

If the present basis is inadequate, the most important point to consider is whether there could be an extra excited one-electron level whose energy lies between those of the highest occupied level $\left(B_{3 u}\right)$ and the lowest excited one $\left(A_{1 g}\right)$ mentioned in $\S 3.2$. If this extra level had $A_{1 g}, B_{1 g}$ or $B_{2 g}$ symmetry, then it could be responsible for the $\pi$ luminescence of the triplet state. Moreover, present experience suggests most of the other triplet states would again have relative energies of about the right value. Further, if this extra one-electron function were mainly on the cations, one could understand why the $\sigma$ luminescence involved recombination from a different state, $\left|\alpha A_{1,} ; \alpha B_{3 u}\right\rangle$ rather than $\left|\alpha X: \alpha \mathrm{B}_{3 u}\right\rangle$.

\section{Conclusions}

Hartree-Fock calculations have been performed for the relaxed exciton in $\mathrm{NaCl}$. The calculations were intended to do two things. The first aim was to produce results 
which could be used as a test for the simpler and less sophisticated approaches that will be necessary in more complex situations. The second aim was to analyse one system for which there are good experimental data, yet where there are still ambiguities of interpretation. A fair degree of success has been achieved in both directions, although problems remain.

The major problem is that, whilst we can understand either the singlet $(\sigma)$ states or the triplet $(\pi)$ states without difficulty, there is conflict when both are considered. This point has not been resolved yet. So far as the other features are concerned, the qualitative features are best described by comparison with the phenomenological models of earlier workers. The quantitative comparisons with experiment are given in table 2 . As stressed by Blair et al we find that the metal ions are important in the excited states. It is not enough to concentrate on the two chlorine ions, as is sometimes argued. However, contrary to Blair et al, the order of the excited states is modified. In particular, the $A_{1 \mathrm{~g}}$ state lies lower than $B_{1 \mathrm{u}}$. In consequence, the results are much closer to those of Kabler's model. For example, the triplet transition is spin-forbidden, not parity forbidden. It is the halogen spin-orbit coupling which renders the triplet transition allowed, not electronlattice coupling. This is in line with experiments on a wide range of the alkali halides. Further, we also find the $\mathrm{V}_{\mathrm{k}}$-like and M-like transitions proposed by Williams and Kabler. Again, there are minor differences in detail. The $V_{k} \pi$ transitions contribute to one of the experimental bands attributed to M-like transitions, although the $V_{k}$ contribution is probably very low in intensity, and our results do not show an $\mathrm{M} \sigma$ transition where expected. But, excepting the problem mentioned earlier, there is substantial agreement between theory and experiment.

One set of measurements which has not been used so far is that of spin resonance in the lowest triplet state (Wasiela et al, Marrone et al). The present calculations have, of course, also produced wavefunctions from which the spin-resonance parameters can be obtained. In general terms, the wavefunctions suggest that broad agreement will be obtained, but that it is unlikely that the extra data can resolve the problem mentioned earlier.

\section{Acknowledgments}

I am indebted to Dr M N Kabler, Dr D Pooley and Dr A E Hughes for valuable discussions. I am particularly grateful to Dr V E Saunders for the use of the ATMOL program.

\section{Appendix. Notation for one-electron orbitals}

We follow the conventions of Blair et al for orbitals of the $(\mathbf{M X})_{2}$ complex. The axes of the complex are labelled as follows:

$$
\begin{array}{ll}
1 \text { (or } y) & \mathrm{M}-\mathrm{M} \text { axis } \\
2(\text { or } z) & \text { Axis normal to the }(\mathrm{MX})_{2} \text { complex } \\
3 \text { (or } x) & \mathrm{X}-\mathrm{X} \text { axis. }
\end{array}
$$

There are six classes of state, which can be related to the states of $\left(\mathrm{X}^{-}\right)_{2}$ when the alkali 

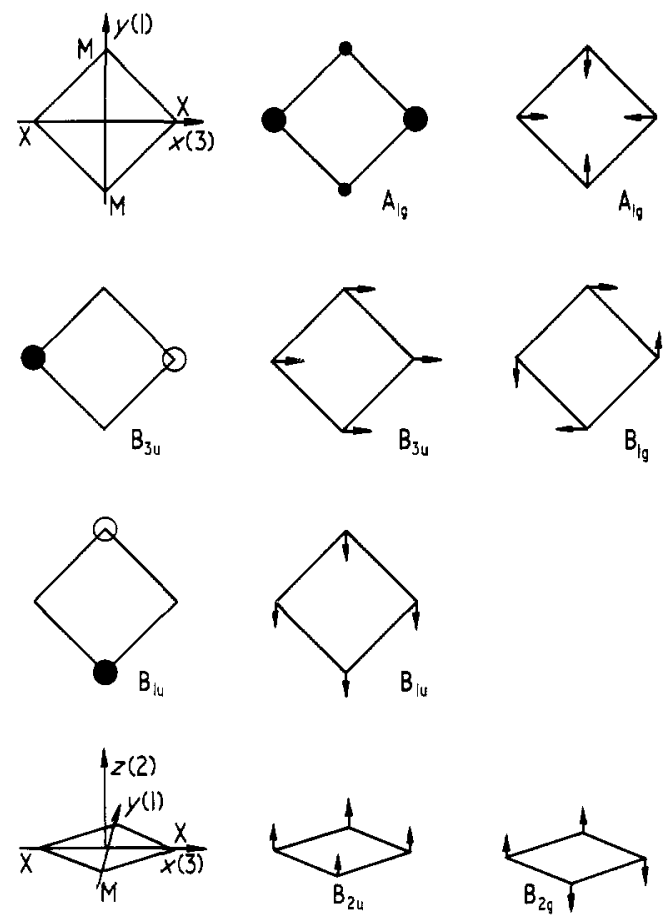

Figure A.1. Symmetry of orbitals. The notation is as follows : (a) for s orbitals, $O$ or where the full circles refer to a term with positive weight and the open circles to a term with negative weight; $(b)$ for $\mathrm{p}$ orbitals, $\rightarrow$. In some cases, more than one independent component is shown on the same diagram: no attempt is made to represent the different weights of the functions.

terms are eliminated. The states are illustrated in figure A.1 for s or p orbitals.

$$
\begin{array}{ll}
\mathbf{A}_{1 \mathrm{~g}} \equiv \sigma & \mathbf{B}_{3 \mathrm{u}} \equiv \sigma_{\mathrm{u}} \\
\mathbf{B}_{1 \mathrm{~g}} \equiv \pi_{y \mathrm{~g}} & \mathbf{B}_{1 \mathrm{u}} \equiv \pi_{y \mathrm{u}} \\
\mathbf{B}_{2 \mathrm{~g}} \equiv \pi_{z \mathrm{~g}} & \mathbf{B}_{2 \mathrm{u}} \equiv \pi_{z \mathrm{u}}
\end{array}
$$

\section{References}

Bartram R H, Stoneham A M and Gash P 1968 Phys. Rev. 176 1014-24

Blair I M, Pooley D and Smith D 1972 J. Phys. C: Solid St. Phys 5 1537-52

Clementi E 1965 IBM J. Res. Dev. 9 Suppl

Fisbach J V, Frohlich D and Kabler M N 1973 J. Luminescence $629-43$

Fowler W B, Marrone M J and Kabler M N 1973 Phys. Rev. B 8 5909-19

Fuller R G, Williams R T and Kabler M N 1970 Phys. Rev. Lett. 25 446-9

Gilbert T L and Wahl A C 1971 J. chem. Phys. 55 5247-61

Jette A N, Das T P and Gilbert T L 1969 Phys. Rev. $184884-94$

Kabler M N 1964 Phys. Rev. 136 A1296-302

Kabler M N, Marrone M J and Fowler W B 1972 Luminescence of Crystals, Molecules and Solutions ed F E Williams (New York: Plenum) pp 171-80

Kabler M N and Patterson D A 1962 Phys. Rev. Lett. $19652-5$

Marrone M J, Patten F W and Kabler M N 1973 Phys. Rev. Lett. 31 467-71

Mott N F and Gurney R W 1948 Electronic Processes in Ionic Crystals (London: Oxford UP)

Murray R B and Keller F J 1965 Phys. Rev. 137 A942-8 
Roessler D M and Walker W C 1968 Phys. Rev. 166 599-606

Wasiela A, Ascarelli G and Merle d'Aubigné 1973a Phys. Rev. Lett. 31 993-6 1973b J. Phys., Paris in press

Williams R T and Kabler M N 1972 Solid St. Commun. 10 49-51 1974 Phys. Rev. B 9 in press

Wood R F 1966 Phys. Rev. $151629-41$ 